\title{
Pharmacogenomics study of thiazide diuretics and QT interval in multi-ethnic populations : the cohorts for heart and aging research in genomic epidemiology
}

Seyerle, A. A.

2018-03

Seyerle , A A , Sitlani , C M , Noordam , R , Gogarten , S M , Li , J , Li , X , Evans , D S , Sun , F , Laaksonen, M A , Isaacs , A, Kristiansson, K, Highland, H M , Stewart, J D , Harris , T B , Trompet , S, Bis , J C, Peloso, G M , Brody , J A, Broer , L, Busch , E L, Duan , Q , Stilp , A M , O'Donnell , C J , Macfarlane , P W , Floyd , J S , Kors , J A , Lin , H J , Li-Gao , R, Sofer , T, Mendez-Giraldez, R, Cummings, S R, Heckbert, S R, Hofman , A, Ford, D , Li , Y , Launer , L J , Porthan , K, Newton-Cheh , C , Napier , M D , Kerr , K F , Reiner , A P , Rice , K M , Roach , J , Buckley, B M , Soliman , E Z, de Mutsert , R , Sotoodehnia , N , Uitterlinden , A G , North , K E \& Chen , Y-D 2018 , ' Pharmacogenomics study of thiazide diuretics and QT interval in multi-ethnic populations : the cohorts for heart and aging research in genomic epidemiology ', Pharmacogenomics Journal , vol. 18 , no. 2 , pp. 215-226 . https://doi.org/10.1038/tpj.2017.10

http://hdl.handle.net/10138/301995

https://doi.org/10.1038/tpj.2017.10

unspecified

publishedVersion

Downloaded from Helda, University of Helsinki institutional repository.

This is an electronic reprint of the original article.

This reprint may differ from the original in pagination and typographic detail.

Please cite the original version. 
ORIGINAL ARTICLE

\title{
Pharmacogenomics study of thiazide diuretics and QT interval in multi-ethnic populations: the cohorts for heart and aging research in genomic epidemiology
}

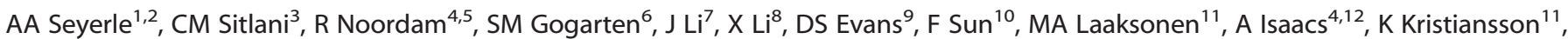

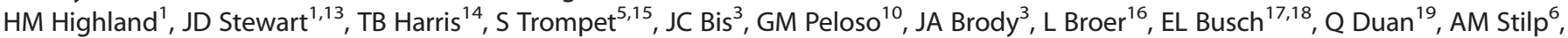 \\ CJ O’Donnell ${ }^{20,21,22}$, PW Macfarlane ${ }^{23}$, JS Floyd ${ }^{3,24}$, JA Kors ${ }^{25}$, HJ Lin ${ }^{8,26}$, R Li-Gao ${ }^{27}$, T Sofer ${ }^{3}$, R Méndez-Giráldez ${ }^{1}$, SR Cummings ${ }^{9}$, \\ SR Heckbert ${ }^{24}$, A Hofman ${ }^{4}$, I Ford ${ }^{28}$, Y Li $^{19,29,30}$, LJ Launer ${ }^{14}$, K Porthan $^{31}$, C Newton-Cheh ${ }^{23,32,33,34}$, MD Napier $^{1}$, KF Kerr $^{6}$, AP Reiner ${ }^{24,35}$, \\ KM Rice ${ }^{6}$, J Roach $^{36}$, BM Buckley ${ }^{37}$, EZ Soliman ${ }^{38}$, R de Mutsert ${ }^{27}$, N Sotoodehnia ${ }^{24,39,40}$, AG Uitterlinden ${ }^{16}$, KE North ${ }^{1}$, CR Lee $^{41}$, \\ V Gudnason ${ }^{42,43}$, T Stürmer ${ }^{1,44}$, FR Rosendaal $^{27}$, KD Taylor $^{8}, \mathrm{KL} \mathrm{Wiggins}^{3}$, JG Wilson ${ }^{45}$, Y-DI Chen ${ }^{8}$, RC Kaplan $^{46}, \mathrm{~K} \mathrm{Wilhelmsen}^{19,47}$, \\ LA Cupples ${ }^{10,21}$, V Salomaa ${ }^{11}$, C van Duijn ${ }^{4}$, JW Jukema ${ }^{15,48,49}$, Y Liu ${ }^{50}$, DO Mook-Kanamori ${ }^{27,51,52}{ }^{\text {, LA Lange }}{ }^{19}$, RS Vasan ${ }^{21,53}$, \\ AV Smith ${ }^{42,43}$, BH Stricker ${ }^{4,54}$, CC Laurie ${ }^{6}$, Jl Rotter ${ }^{8}$, EA Whitsel ${ }^{1,55}$, BM Psaty ${ }^{3,24,56,57}$ and CL Avery ${ }^{1,13}$
}

Thiazide diuretics, commonly used antihypertensives, may cause QT interval (QT) prolongation, a risk factor for highly fatal and difficult to predict ventricular arrhythmias. We examined whether common single-nucleotide polymorphisms (SNPs) modified the association between thiazide use and QT or its component parts (QRS interval, JT interval) by performing ancestry-specific, transethnic and cross-phenotype genome-wide analyses of European (66\%), African American (15\%) and Hispanic (19\%) populations $(N=78$ 199), leveraging longitudinal data, incorporating corrected standard errors to account for underestimation of interaction estimate variances and evaluating evidence for pathway enrichment. Although no loci achieved genome-wide significance $\left(P<5 \times 10^{-8}\right)$, we found suggestive evidence $\left(P<5 \times 10^{-6}\right)$ for SNPs modifying the thiazide-QT association at 22 loci, including ion transport loci (for example, NELL1, KCNQ3). The biologic plausibility of our suggestive results and simulations demonstrating modest power to detect interaction effects at genome-wide significant levels indicate that larger studies and innovative statistical methods are warranted in future efforts evaluating thiazide-SNP interactions.

The Pharmacogenomics Journal (2018) 18, 215-226; doi:10.1038/tpj.2017.10; published online 18 July 2017

\footnotetext{
'Department of Epidemiology, University of North Carolina, Chapel Hill, NC, USA; ${ }^{2}$ Division of Epidemiology and Community Health, University of Minnesota, Minneapolis, MN, USA; ${ }^{3}$ Department of Medicine, University of Washington, Seattle, WA, USA; ${ }^{4}$ Department of Epidemiology, Erasmus MC—University Medical Center Rotterdam, Rotterdam, The Netherlands; ${ }^{5}$ Department of Gerontology and Geriatrics, Leiden University Medical Center, Leiden, The Netherlands; ${ }^{6}$ Department of Biostatistics, University of Washington, Seattle, WA, USA; ${ }^{7}$ Department of Medicine, Division of Cardiovascular Medicine, Stanford University School of Medicine, Palo Alto, CA, USA; ${ }^{8}$ Institute for Translational Genomics and Population Sciences, Department of Pediatrics, Los Angeles Biomedical Research Institute at Harbor-UCLA Medical Center, Torrance, CA, USA; ${ }^{9}$ California Pacific Medical Center Research Institute, San Francisco, CA, USA; ${ }^{10}$ Department of Biostatistics, Boston University School of Public Health, Boston, MA, USA; ${ }^{11}$ Department of Health, THL-National Institute for Health and Welfare, Helsinki, Finland; ${ }^{12}$ CARIM School of Cardiovascular Diseases, Maastricht Centre for Systems Biology (MaCSBio), and Department of Biochemistry, Maastricht University, Maastricht, The Netherlands; ${ }^{13}$ Carolina Population Center, University of North Carolina, Chapel Hill, NC, USA; ${ }^{14}$ Laboratory of Epidemiology, Demography, and Biometry, National Institute on Aging, Bethesda, MD, USA; ${ }^{15}$ Department of Cardiology, Leiden University Medical Center, Leiden, The Netherlands; ${ }^{16}$ Department of Internal Medicine, Erasmus MC-University Medical Center Rotterdam, Rotterdam, The Netherlands; ${ }^{17}$ Channing Division of Network Medicine, Department of Medicine, Brigham and Women's Hospital and Harvard Medical School, Boston, MA, USA; ${ }^{18}$ Department of Epidemiology, Harvard T.H. Chan School of Public Health, Boston, MA, USA; ${ }^{19}$ Department of Genetics, University of North Carolina, Chapel Hill, NC, USA; ${ }^{20}$ Department of Medicine, Harvard University, Boston, MA, USA; ${ }^{21}$ National Heart, Lung, and Blood Institute Framingham Heart Study, Framingham, MA, USA; ${ }^{22}$ Cardiology Section, Boston Veterans Administration Healthcare, Boston, MA, USA; ${ }^{23}$ Institute of Health and Wellbeing, University of Glasgow, Glasgow, UK; ${ }^{24}$ Department of Epidemiology, University of Washington, Seattle, WA, USA; ${ }^{25}$ Department of Medical Informatics, Erasmus MC-University Medical Center Rotterdam, Rotterdam, The Netherlands; ${ }^{26}$ Division of Medical Genetics, Department of Pediatrics, Harbor-UCLA Medical Center, Torrance, CA, USA; ${ }^{27}$ Department of Clinical Epidemiology, Leiden University Medical Center, Leiden, The Netherlands; ${ }^{28}$ Robertson Center for Biostatistics, University of Glasgow, Glasgow, UK; ${ }^{29}$ Department of Biostatistics, University of North Carolina, Chapel Hill, NC, USA; ${ }^{30}$ Department of Computer Science, University of North Carolina, Chapel Hill, NC, USA; ${ }^{31}$ Division of Cardiology, Heart and Lung Center, Helsinki University Central Hospital, Helsinki, Finland; ${ }^{32}$ Center for Human Genetic Research, Massachusetts General Hospital, Boston, MA, USA; ${ }^{33}$ Program in Medical and Population Genetics, Broad Institute, Cambridge, MA, USA; ${ }^{34}$ Cardiovascular Research Center, Massachusetts General Hospital, Boston, MA, USA; ${ }^{35}$ Public Health Sciences Division, Fred Hutchinson Cancer Research Center, Seattle, WA, USA; ${ }^{36}$ Research Computing Center, University of North Carolina, Chapel Hill, NC, USA; ${ }^{37}$ Department of Pharmacology and Therapeutics, University College Cork, Cork, Ireland; ${ }^{38}$ Epidemiology Cardiology Research Center (EPICARE), Wake Forest School of Medicine, Winston-Salem, NC, USA; ${ }^{39}$ Division of Cardiology, University of Washington, Seattle, WA, USA; ${ }^{40}$ Cardiovascular Health Research Unit, Department of Medicine, University of Washington, Seattle, WA, USA; ${ }^{41}$ Division of Pharmacotherapy and Experimental Therapeutics, Eshelman School of Pharmacy, University of North Carolina, Chapel Hill, NC, USA; ${ }^{42}$ Icelandic Heart Association, Kopavogur, Iceland; ${ }^{43}$ Department of Medicine, University of Iceland, Reykjavik, Iceland; ${ }^{44}$ Center for Pharmacoepidemiology, University of North Carolina, Chapel Hill, NC, USA; ${ }^{45}$ Department of Physiology and Biophysics, University of Mississippi Medical Center, Jackson, MS, USA; ${ }^{46}$ Department of Epidemiology and Population Health, Albert Einstein College of Medicine, Bronx, NY, USA; ${ }^{47}$ The Renaissance Computing Institute, Chapel Hill, NC, USA; ${ }^{48}$ Durrer Center for Cardiogenetic Research, Amsterdam, The Netherlands; ${ }^{49}$ Interuniversity Cardiology Institute of the Netherlands, Utrecht, The Netherlands; ${ }^{50}$ Department of Epidemiology and Prevention, Division of Public Health Sciences, Wake Forest University, Winston-Salem, NC, USA; ${ }^{51}$ Department of Public Health and Primary Care, Leiden University Medical Center, Leiden, the Netherlands; ${ }^{52}$ Department of BESC, Epidemiology Section, King Faisal Specialist Hospital and Research Centre, Riyadh, Saudi Arabia; ${ }^{53}$ Division of Preventive Medicine and Epidemiology, Department of Epidemiology, Boston University School of Medicine, Boston, MA, USA; ${ }^{54}$ Inspectorate of Health Care, Utrecht, The Netherlands; ${ }^{55}$ Department of Medicine, University of North Carolina, Chapel Hill, NC, USA; ${ }^{56}$ Department of Health Services, University of Washington, Seattle, WA, USA and ${ }^{57}$ Group Health Research Institute, Group Health Cooperative, Seattle, WA, USA. Correspondence: Dr A Seyerle, Department of Epidemiology and Community Health, University of Minnesota, 1300 S 2nd Street, Suite 300, Minneapolis, MN 55454, USA. E-mail: aseyerle@umn.edu

Received 20 July 2016; revised 14 January 2017; accepted 9 March 2017; published online 18 July 2017
} 


\section{INTRODUCTION}

Over the past decade, the use of prescription drugs has skyrocketed, with nearly half of all Americans now taking at least one prescription drug. ${ }^{1}$ Accompanying the increased prevalence of drug use is a high burden of adverse drug reactions (ADRs), which account for approximately 100000 deaths and 2.2 million serious health effects annually. ${ }^{2-4}$ QT interval (QT) prolongation, which can trigger fatal ventricular arrhythmias, is a longrecognized adverse effect ${ }^{5}$ of numerous common medications, such as antipsychotics, antibiotics, antiarrhythmics and antihypertensives. ${ }^{6}$ Within the past ten years, QT prolongation has represented the most common cause for withdrawal of a drug from the market (or relabeling) after approval by the US Food and Drug Administration (FDA). ${ }^{7,8}$ However, drug-induced QT prolongation remains difficult to predict. $^{9}$

Genetic variants are known to mediate both pharmacokinetic and pharmacodynamic processes, thereby playing a major role in drug response. ${ }^{10}$ Pharmacogenomics, which evaluates the role of genetics in drug response, offers a promising avenue for understanding variation in drug response, ${ }^{11}$ illuminating novel pathways, informing drug development and selection, ${ }^{12-14}$ optimizing dosing regimens ${ }^{15-19}$ and avoiding ADRs. ${ }^{20-22}$ QT is highly heritable (35-40\%). ${ }^{23-27}$ Previous pharmacogenomics studies of drugs associated with QT prolongation, including thiazide diuretics, a common anti-hypertensive therapy used by over a quarter of the United States hypertensive population, ${ }^{28}$ identified multiple loci associated with anti-hypertensive response and ADRs. ${ }^{29-34}$ Furthermore, thiazide diuretics are used unequally across race/ethnic groups in the United States, with approximately $10 \%$ of Hispanic/Latinos, $13 \%$ of European Americans, and $23 \%$ of African Americans taking a thiazide diuretic. ${ }^{28,35,36}$ Therefore, the pharmacogenomics of thiazide-induced QT prolongation represents an excellent but understudied candidate for pharmacogenomic inquiry.

We previously examined evidence for common singlenucleotide polymorphisms (SNPs) that modified the association between thiazide use and QT and failed to identify any genomewide significant loci $\left(P<5 \times 10^{-8}\right) .{ }^{37}$ However, our previous study was limited to European descent populations and cross-sectional analyses, despite many of the contributing studies having longitudinal drug and electrocardiographic data. ${ }^{37}$ Here, we expand upon that work, applying recent statistical innovations to leverage longitudinal data and including an additional 44418 participants of European, African American, and Hispanic/Latino descent to perform the first trans-ethnic genome-wide association study (GWAS) to examine genetic associations that modify the association between thiazides and QT, as well as the component parts of QT (JT interval (JT), QRS interval (QRS)).

\section{MATERIALS AND METHODS}

\section{Study populations}

Fourteen cohorts from in the Cohorts for Heart and Aging Research in Genomic Epidemiology (CHARGE) ${ }^{38}$ Pharmacogenomics Working Group (PWG) participated in this analysis, contributing 78199 participants: European descent (51 601), African American (11 482), and Hispanic/Latino (15 116) participants (Table 1; Supplementary Text). Among the fourteen cohorts, six (55\% of the total population) had repeated measurements of medication use and electrocardiogram (ECG) assessments and contributed longitudinal data to the analysis: Age, Gene/Environment SusceptibilityReykjavik Study (AGES), Atherosclerosis Risk in Communities (ARIC) Study, Cardiovascular Health Study (CHS), Rotterdam Study (RS), Multi-Ethnic Study of Atherosclerosis (MESA), and Women's Health Initiative (WHI). The remaining eight cohorts contributed cross-sectional data to the analysis: Framingham Heart Study (FHS), Erasmus Rucphen Family (ERF) Study, Health 2000 (H2000), Health, Aging, and Body Composition (Health ABC), Prospective Study of Pravastatin in the Elderly at Risk (PROSPER), Jackson Heart Study (JHS), Netherlands Epidemiology of Obesity (NEO) Study, and Hispanic Community Health Study/Study of Latinos (HCHS/SOL).

\section{Study design}

Participants with ECG measurements, medication assessment, and genome-wide genotype data were eligible for inclusion. The following exclusion criteria were applied: poor ECG quality, atrial fibrillation detected by ECG, pacemaker implantation, second or third degree atrioventricular heart block, QRS greater than $120 \mathrm{~ms}$, prevalent heart failure, pregnancy, missing ECG, missing medication assessment, missing genotype information, or race/ethnicity other than European descent, African American, or Hispanic/Latino. For studies with longitudinal data, exclusion criteria were applied on a visit-specific basis.

\section{Medication assessment}

Medication use was assessed through medication inventories conducted during clinic visits, home interviews, or through pharmacy databases (Supplementary Table 1). Six studies captured medication used on the day of the study visit. A further six of the 14 participating cohorts captured medications used 1-2 weeks preceding ECG assessment. HCHS/SOL ascertained medications used within four weeks preceding ECG measurement, and the RS captured medication used within 30 days preceding ECG assessment. Participants were classified as thiazide diuretic users if they took a thiazide or thiazide-like diuretic in a single or combination preparation, with or without potassium (K)-sparing agents, and with or without K-supplements.

For cross-sectional studies, the number of exposed participants ( $\left.N_{\text {exposed }}\right)$ was defined as the number of participants classified as thiazide users. For studies with longitudinal data, $N_{\text {exposed }}$ was calculated as follows:

$$
N_{\text {exposed }}=\sum_{i} \frac{n_{i}}{1+\left(n_{i}-1\right) \hat{\rho}} \frac{\#\left\{E_{i t}=1\right\}}{n_{i}}
$$

where $\mathrm{n}_{i}$ is the number of observations for participant $i, \hat{\rho}$ is an estimate of the pairwise visit-to-visit correlation within participants from a Generalized Estimating Equation (GEE)-exchangeable model that does not contain genetic data, and $\#\left\{E_{i t}=1\right\}$ is the number of observations for which participant $i$ was exposed. ${ }^{39}$

\section{ECG interval measurement}

QT and QRS were digitally recorded by each participating study using resting, supine or semi-recumbent, standard 12-lead ECGs (Supplementary Table 2). Comparable procedures were used for preparing participants, placing electrodes, recording, transmitting, processing, and controlling quality of ECGs. Studies used Marquette MAC 5000, MAC 12, MAC 1200 or MAC PC (GE Healthcare, Milwaukee, Wisconsin, USA), University of Glasgow (Cardiac Science, Manchester, UK), or ACTA (EASOTE, Florence, Italy) machines. Recordings were processed using one of the following programs (Marquette 12SL, MEANS, University of Glasgow, Digital Calipers, or Health 2000 custom-made software. JT was calculated by the formula: $\mathrm{JT}=\mathrm{QT}-\mathrm{QRS}$.

\section{Genotyping and imputation}

Each study conducted genome-wide genotyping independently using either Affymetrix (Santa Clara, CA, USA) or Illumina (San Diego, CA, USA) arrays (Supplementary Table 3). Sex mismatches, duplicate samples, and first-degree relatives (except in ERF, FHS, HCHS/SOL and JHS) were excluded. DNA samples with call rates less than $95-98 \%$ were excluded, as were SNPs with SNP call rates less than 90-98\%, minor allele frequencies (MAF) less than $1 \%$, or that failed Hardy-Weinberg equilibrium. To maximize genome coverage and comparisons across genotyping platforms, genotypes were imputed using HapMap2, ${ }^{40-42} 1000$ Genomes Phase 1, or 1000 Genomes Phase 3 reference panels. ${ }^{43,44}$ Genotypes imputed using build 37 were lifted over to build $36^{45,46}$ to enable comparisons between imputation platforms and results were restricted to SNPs present in HapMap2.

\section{Statistical analyses}

Genome-wide pharmacogenomic analyses were performed by each cohort independently across $\sim 2.5$ million SNPs for QT, QRS, and JT separately. Drug-SNP interactions were estimated assuming an additive genetic model, using mixed effect models, GEE, or linear regression with robust standard errors. The analytic model varied based on study design and the availability of longitudinal data (Supplementary Table 4). All analyses were adjusted for age (years), sex when applicable, study site or region, principal 
Table 1. Study population characteristics of 25 contributing study populations

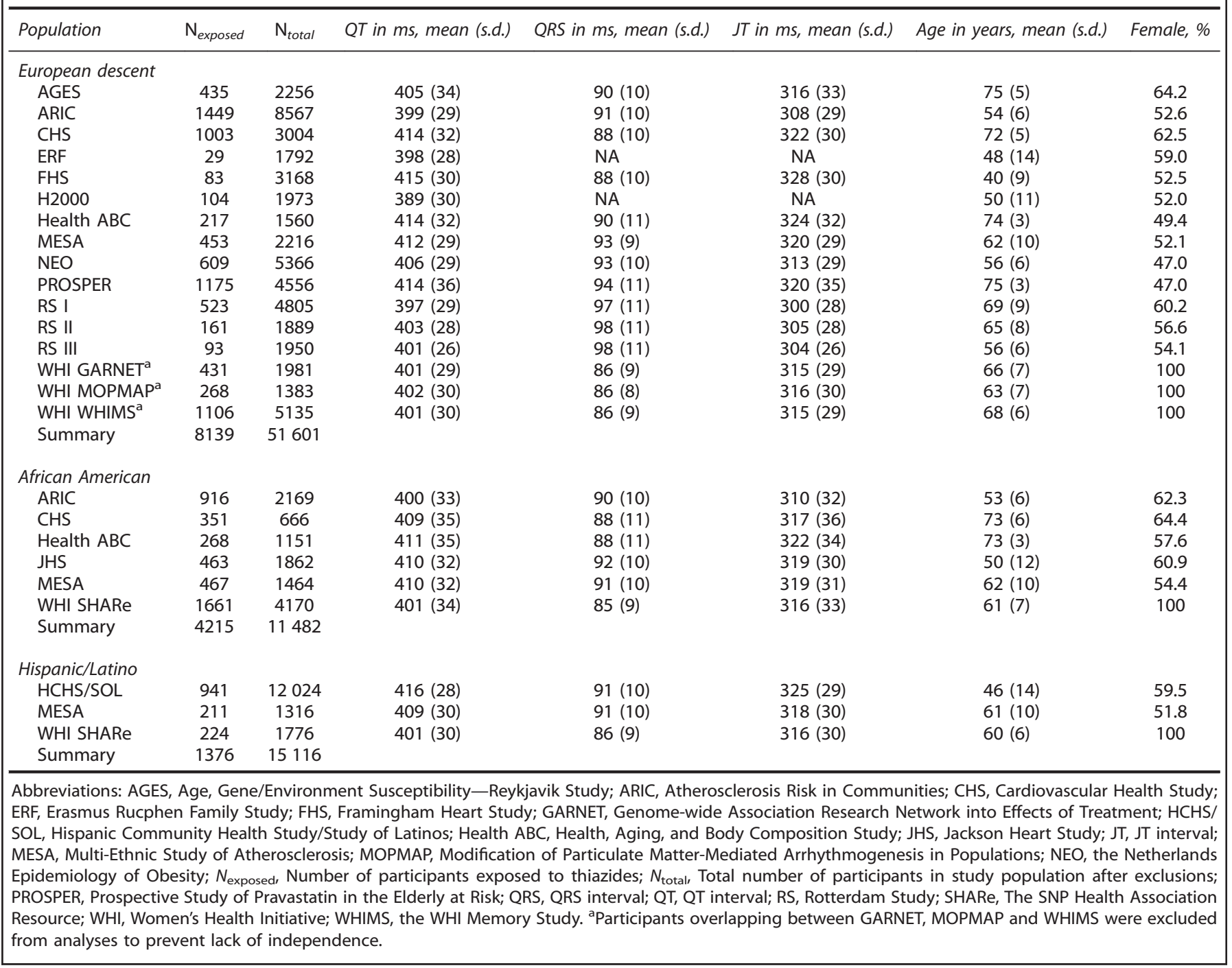

components of genetic ancestry, visit-specific RR interval (ms), and visitspecific QT altering medications defined using the University of Arizona Center for Education and Research on Therapeutics (UAZ CERT) QT-prolonging drug classification. ${ }^{6}$ Furthermore, ERF, FHS and HCHS/SOL incorporated estimates of relatedness into all analyses. Study-specific results were corrected for genomic inflation $(\lambda)$.

Previous simulations demonstrated that models using robust standard errors underestimate the variance of coefficient estimates for SNPs with low MAFs. ${ }^{39}$ To account for this underestimation, corrected standard errors were calculated using a (Student's) $t$-reference distribution. ${ }^{39}$ The degrees of freedom (df) for the $t$-reference distribution were estimated using Satterthwaite's method. ${ }^{47}$ When cohorts were unable to implement Satterthwaite's method, an approximate df was calculated as twice the cohort- and SNP-specific product of the SNP imputation quality (range: $0,1)$, the MAF (range: $0.0,0.50$ ), and $N_{\text {exposed. }}$ Standard errors were then 'corrected' by assuming a normal reference distribution that yielded the $t$-distribution based $P$-values from the beta estimates. ${ }^{39}$ Furthermore, because simulations demonstrated that corrected standard errors were unstable when minor allele counts among the exposed were low, a cohortspecific df filter of 15 was applied across all SNPs. ${ }^{39}$

For each trait, race-stratified and trans-ethnic betas and corrected standard errors were combined with inverse-variance weighted metaanalysis conducted in METAL. ${ }^{48}$ We used a genome-wide significance threshold of $P<5 \times 10^{-8}$ and a suggestive threshold of $P<5 \times 10^{-6}$. However, the assumptions of a fixed-effects meta-analysis do not always hold between race/ethnicities due to differences in patterns of linkage disequilibrium (LD) across ancestral populations, potential allelic heterogeneity, differences in gene-environment and gene-gene interactions, and differences in environmental and lifestyle factors. ${ }^{49,50}$ Therefore, transethnic meta-analysis was also conducted using the Bayesian MANTRA approach and a genome-wide threshold of $\log _{10}$ (Bayes Factor (BF)) $>6$ and a suggestive threshold of $\log _{10}(\mathrm{BF})>5.51$ Additionally, previous studies have demonstrated the potential to increase power and detect evidence of pleiotropy by conducting multi-trait analysis across correlated traits. ${ }^{52,53}$ To examine potential pleiotropy across ventricular depolarization and repolarization, we conducted cross-phenotype meta-analysis combining $t$-statistics across QRS and JT using an adaptive sum of powered score (aSPU) test, which tests for both concordant and discordant associations across some or all of the included traits. ${ }^{54}$ The reference distribution for the aSPU test was calculated using $10^{8}$ simulations.

Genome-wide significant and suggestive meta-analysis results were examined for gene or pathway enrichment. Previous work has shown that it is beneficial to apply multiple methods of gene-set analysis (GSA) when the underlying etiology of the genetic mechanism is unclear. ${ }^{55-57}$ We therefore used two methods of GSA. We performed a multiple regression gene analysis approach followed by a self-contained GSA using gene-level regression as implemented in MAGMA. ${ }^{58}$ Post-meta-analysis $P$-values were used as input in the analysis and gene-sets were collected from Ingenuity, ${ }^{59}$ Panter, ${ }^{60} \mathrm{KEGG}^{61}$ and ConsensusPathDB ${ }^{62,63}$ and restricted to biologically motivated pathways involved in the following: ion transport 
and homeostasis, transcription and translation, renal and cardiac development and function, and pharmacokinetic/dynamic pathways. Additionally, we selected all SNPs with $P<1 \times 10^{-5}$ for analysis with DEPICT, which searches for gene, gene-set and tissue enrichment among 14461 reconstituted gene-sets, eliminating the need to select candidate genesets. ${ }^{64}$ To account for multiple testing, we applied a false discovery rate (FDR) threshold of $5 \%$ for both GSA approaches.

\section{Statistical power simulations}

Statistical power to detect drug-SNP interactions using cross-sectional and longitudinal modeling approaches was estimated via simulation studies. Assumptions, which were informed by European ancestry populations, included: (1) 50000 participants; (2) a two-sided, per-SNP $a=5 \times 10^{-8}$; (3) a mean heart rate-corrected QT (standard deviation) $=400$ (30) ms; (4) $N_{\text {exposed }}=8100 ;(5)$ a mean drug effect for those with zero copies of the minor allele $=5 \mathrm{~ms}$; (6) a mean SNP effect for those not exposed to drug $=0 \mathrm{~ms}$; (7) a MAF $=0.05$ or 0.25 ; (8) an additive model of inheritance; (9) two study visits for longitudinal simulations; (10) within-person QT correlation $=0.80 ;(11)$ an attrition rate between visits for longitudinal simulations $=0.13 ;(12)$ random missingness rate across study visits $=0.09$; and (13) an independent GEE correlation structure for longitudinal simulations. For longitudinal simulations, drug use was either temporally constant or variable. When variable, drug exposure was assumed to be completely random at both visits.

\section{RESULTS}

Study characteristics

A total of 78199 participants were included in the analysis, of which 13730 (18\%) were exposed to thiazides (Table 1). Thiazide use was most common among African Americans (36\%), compared with $16 \%$ and $9 \%$ among European descent and Hispanic/Latino populations, respectively. Mean age ranged from 40 (FHS) to 75 years (PROSPER) and the percentage of females ranged from $47 \%$ (NEO, PROSPER) to $100 \%$ (WHI). Average QT was between $389 \mathrm{~ms}$ (H2000) and $416 \mathrm{~ms}$ (HCHS/SOL).

Genome-wide analysis of thiazide-SNP interaction and QT interval Q-Q plots for individual study results, as well as for meta-analyzed results, demonstrated adequate calibration of study-specific test statistics (Supplementary Figures 1-4). However, the family-based studies (ERF, FHS, HCHS/SOL) showed modest evidence of over-dispersion $(\lambda=1.07-1.16)$.

No genome-wide significant thiazide-SNP interaction effects were detected in any race/ethnic group (Figure 1). However, suggestive interaction effects $\left(P<5 \times 10^{-6}\right)$ were found for 22 loci in at least one race/ethnic group: European descent (seven loci), African American (six loci), Hispanic/Latino (six loci), or trans-ethnic (nine loci) (Figure 1; Table 2). Only the DNAH8/BTBD9 locus was suggestively significant in more than one race/ethnic group (rs862433 in African Americans, rs1950398 in Hispanic/Latinos). Only two of the suggestive SNPs were heterogeneous across populations with $P_{\text {het }}<0.05$ ( $r$ 4890550 and rs13223427).

Additionally, examination of 35 loci previously associated with QT in a published main effects GWAS $^{65}$ found no significant associations in European descent populations using a Bonferroni corrected threshold of $P<0.001 \quad(0.001=0.05 / 35$; Supplementary Table 5). The magnitude of the interaction effect was close to zero for all but six of the 35 SNP, which had interaction effects greater than $0.50 \mathrm{~ms}$.

Similarly, while no locus showed genome-wide significance in our trans-ethnic MANTRA analysis (Supplementary Figure 5), one SNP (rs2765279) was above the suggestive threshold, with a $\log _{10}(\mathrm{BF})$ of 5.2. Rs2765279, located in RGSL1, a gene involved in G-protein signaling regulation, was also the most significant SNP in the fixed-effects trans-ethnic analysis $\left(P=3 \times 10^{-7}\right)$.
Genome-wide analysis of thiazide-SNP interaction and QRS interval or JT interval

Results for QRS showed a similar pattern to those for QT (Supplementary Figure 6 and Supplementary Table 6). Whereas no results achieved genome-wide significance, 28 loci showed suggestive evidence of modifying the thiazide-QRS association (four loci in European descent populations, 11 in African Americans, eight in Hispanic/Latinos, and seven in trans-ethnic populations) and only one SNP had a $P_{\text {het }}<0.05$ (rs11591185). The most significant SNP, rs7638855 $\left(P=2 \times 10^{-7}\right)$, located upstream from GAP43, was also suggestively significant after trans-ethnic analysis in MANTRA $\left(\log _{10}(B F)=5.4\right.$; Supplementary Figure 5).

Similarly, no SNPs showed genome-wide significant interaction for JT, although 19 loci were suggestively associated (five loci in European descent populations, four in African Americans, five in Hispanic/Latino, and seven in trans-ethnic populations; Supplementary Figure 6 and Supplementary Table 7). No SNPs showed significant heterogeneity between populations. Moreover, MANTRA analysis identified two SNPs that achieved suggestive significance (Supplementary Figure 5). The rs1264878 variant near KCNIP4, a voltage-gated potassium channel interacting protein was the most significant SNP in our fixed-effects meta-analyses $\left(P=3 \times 10^{-7}\right)$ and had a $\log _{10}(B F)=5.1$. However, most significant SNP in MANTRA meta-analyses was rs9303589, in CA10, with a $\log _{10}(B F)=5.1$.

\section{Cross-phenotype meta-analysis}

Cross-phenotype meta-analysis found no genome-wide significant evidence of pleiotropy across QRS and JT (Figure 2 and Supplementary Figure 7). However, eight loci had a suggestive evidence of thiazide-SNP interaction after meta-analyzing QRS and JT results (Table 3 ). These included three loci that were nominally associated with QRS and JT $(P<0.05)$, but whose effects did not reach the suggestive association threshold in either univariate analysis (rs1295230 [PIK3R6], rs6931354 [ADGRB3] and rs8119517 [PREX1]).

Gene and pathway enrichment analysis

Although analysis with DEPICT found no enrichment in a single gene or tissue, gene-set enrichment analysis in European descent populations found enrichment in the ATXN3 subnetwork for the interactive effect of genotype and thiazide use on QT $\left(P=1 \times 10^{-6}\right)$. There was no enrichment found in QRS or JT analyses. MAGMA analyses found significant enrichment in six genes among African Americans in the interactive effect of genotype and thiazide use on QRS: CNTRL, CPN1, FAM65B, RAB14, ISY1, NELL1 (Supplementary Table 8). No other MAGMA analyses found gene enrichment. MAGMA GSA for QT and JT analyses found significant enrichment for transcription and translational pathways, although no gene-set enrichment was found in QRS analyses (Table 4).

\section{Statistical power}

Given the biologic plausibility of the suggestive results for all three traits, we examined statistical power for our analysis to assess our ability to detect interaction effects. Simulations demonstrated that all analyses were underpowered to detect thiazide-SNP interaction effects less than $3 \mathrm{~ms}$ (e.g. 15\% power to detect an interactive effect of $2 \mathrm{~ms}$; Figure 3). However, even with time-varying drug exposure (that is, observed QT measurement on and off drug within an individual), which demonstrated the greatest power, analyses for SNPs with MAF $=5 \%$ did not achieve $80 \%$ power until the thiazide-SNP interaction effect reached $6 \mathrm{~ms}$. 

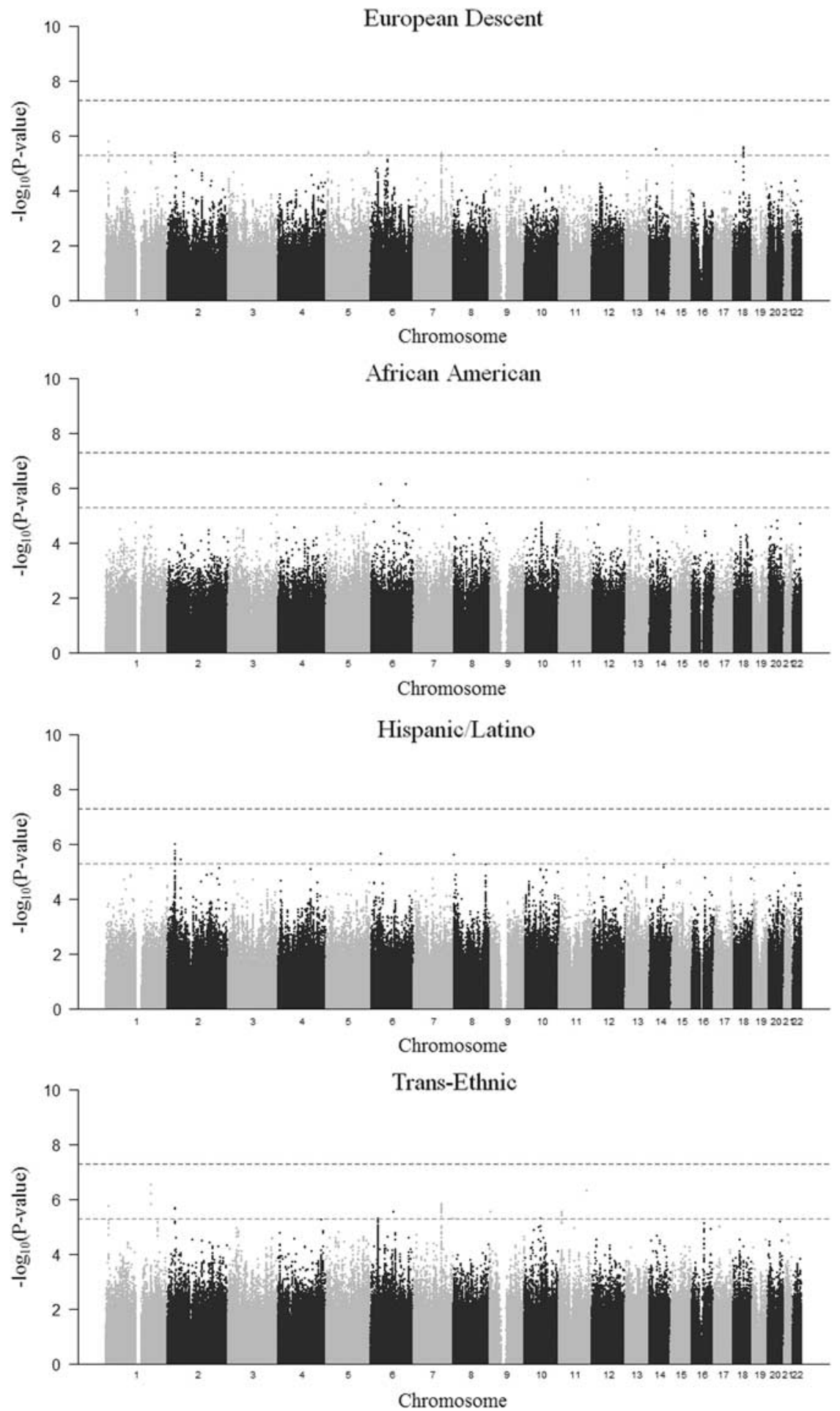

Figure 1. Manhattan plots of $P$-values for thiazide-SNP (single-nucleotide polymorphism) interaction estimates for QT interval analyses after fixed-effects meta-analysis among European descent populations $(N=51601)$, African American populations $(N=11482)$, Hispanic/Latino populations $(N=15116)$, and all populations (trans-ethnic). Each study was analyzed using linear regression, mixed-effects models, or generalized estimating equations and SNPs with a study-specific degree of freedom measure (df = twice the cohort- and SNP-specific product of the SNP imputation quality (range: 0,1 ), the MAF (range: $0.0,0.50$ ), and the number of individuals exposed to thiazide $\left.\left(N_{\text {exposed }}\right)\right)<15$ were excluded from meta-analysis. The $x$-axis represents the chromosomal position and the $y$-axis represents the $-\log _{10}(P$-value $)$. On each plot, genome-wide significance $\left(P<5 \times 10^{-8}\right)$ and suggestive significance $\left(P<5 \times 10^{-6}\right)$ are denoted with dashed lines.

\section{DISCUSSION}

In this study, we examined 78199 participants of European, African American or Hispanic/Latino descent for evidence of
thiazide-SNP interactions influencing QT. Although we used a comprehensive approach that considered multi-ethnic populations, leveraged pleiotropy, accommodated population 
Table 2. Loci with suggestive evidence of association with the thiazide-SNP interaction effect on QT interval

\begin{tabular}{|c|c|c|c|c|c|c|c|c|}
\hline Locus & $S N P$ & Chr & Position $^{\mathrm{a}}$ & $C A$ & $C A F$ & Interaction effect in ms (s.e.) & P-value & $\mathrm{P}_{\text {het }}$ \\
\hline \multicolumn{9}{|c|}{ European descent } \\
\hline KIAA2013 & rs17367934 & 1 & 11890791 & $A$ & 0.89 & $2.4(0.5)$ & $2 \times 10^{-6}$ & 0.9 \\
\hline$S L C 14 A 2$ & rs4890550 & 18 & 41409189 & C & 0.44 & $-1.4(0.3)$ & $3 \times 10^{-6}$ & 0.01 \\
\hline RPS 29 & rs10143493 & 14 & 47999650 & A & 0.01 & $-10.6(2.3)$ & $3 \times 10^{-6}$ & 0.4 \\
\hline NELL1 1 & rs12225793 & 11 & 21057283 & $\mathrm{~T}$ & 0.12 & $2.3(0.5)$ & $4 \times 10^{-6}$ & 1.0 \\
\hline STC2 & rs10079004 & 5 & 172704698 & A & 0.71 & $-1.5(0.3)$ & $4 \times 10^{-6}$ & 0.4 \\
\hline LCLAT1 & rs7608507 & 2 & 30447424 & A & 0.75 & $1.6(0.3)$ & $4 \times 10^{-6}$ & 0.7 \\
\hline PPP1R3A & rs13223427 & 7 & 113199332 & $\mathrm{~T}$ & 0.56 & $1.4(0.3)$ & $4 \times 10^{-6}$ & 0.02 \\
\hline \multicolumn{9}{|c|}{ African American } \\
\hline ZBTB16 & rs10789991 & 11 & 113424299 & $\mathrm{~T}$ & 0.03 & $12.3(2.4)$ & $5 \times 10^{-7}$ & 0.6 \\
\hline DNAH8 & rs862433 & 6 & 38968057 & A & 0.25 & $-2.6(0.5)$ & $7 \times 10^{-7}$ & 0.2 \\
\hline Intergenic & rs9376483 & 6 & 140352934 & $\mathrm{~T}$ & 0.94 & $7.2(1.4)$ & $7 \times 10^{-7}$ & 0.5 \\
\hline CASP8AP2 & rs7753194 & 6 & 90597484 & $A$ & 0.02 & $-11.4(2.4)$ & $3 \times 10^{-6}$ & 0.2 \\
\hline EBF1 & rs11135035 & 5 & 157833407 & A & 0.41 & $2.1(0.5)$ & $4 \times 10^{-6}$ & 0.9 \\
\hline LAMA4 & rs6926485 & 6 & 112630302 & $\mathrm{~T}$ & 0.64 & $2.4(0.5)$ & $5 \times 10^{-6}$ & 0.5 \\
\hline \multicolumn{9}{|c|}{ Hispanic/Latino } \\
\hline SPDYA & rs12475612 & 2 & 28883510 & $\mathrm{~T}$ & 0.48 & $-3.5(0.7)$ & $1 \times 10^{-6}$ & 0.9 \\
\hline BTBD9 & rs1950398 & 6 & 38666897 & $\mathrm{~T}$ & 0.97 & $9.6(2.0)$ & $2 \times 10^{-6}$ & 0.05 \\
\hline$T D R P$ & rs6558894 & 8 & 480495 & $\mathrm{C}$ & 0.14 & $-4.9(1.0)$ & $2 \times 10^{-6}$ & 0.3 \\
\hline COLCA2 & rs10749974 & 11 & 110696967 & A & 0.09 & $-6.0(1.3)$ & $3 \times 10^{-6}$ & 0.2 \\
\hline CRYGGP & rs17868255 & 2 & 51884417 & $A$ & 0.97 & $10.3(2.2)$ & $3 \times 10^{-6}$ & 0.5 \\
\hline RYR3 & rs16968694 & 15 & 31376213 & A & 0.18 & $4.5(1.0)$ & $3 \times 10^{-6}$ & 1.0 \\
\hline \multicolumn{9}{|l|}{ Trans-Ethnic } \\
\hline RGSL1 & rs2765279 & 1 & 180693520 & $\mathrm{~T}$ & 0.28 & $1.4(0.3)$ & $3 \times 10^{-7}$ & 0.4 \\
\hline ZBTB16 & rs10789991 & 11 & 113424299 & $\mathrm{~T}$ & 0.03 & $12.3(2.4)$ & $5 \times 10^{-7}$ & 0.6 \\
\hline PPP1R3A & rs17619887 & 7 & 113142601 & A & 0.47 & $1.2(0.3)$ & $2 \times 10^{-6}$ & 0.07 \\
\hline KIAA2013 & rs17367934 & 1 & 11890791 & A & 0.89 & $2.3(0.5)$ & $2 \times 10^{-6}$ & 1.0 \\
\hline LCLAT1 & rs6756908 & 2 & 30446501 & A & 0.65 & $1.3(0.3)$ & $2 \times 10^{-6}$ & 0.5 \\
\hline FAR1 & rs7130476 & 11 & 13711632 & $\mathrm{C}$ & 0.90 & $2.0(0.4)$ & $3 \times 10^{-6}$ & 0.5 \\
\hline CASP8AP2 & rs7753194 & 6 & 90597484 & A & 0.02 & $-11.4(2.4)$ & $3 \times 10^{-6}$ & 0.2 \\
\hline SMARCA2 & rs1886261 & 9 & 2163590 & A & 0.75 & $1.5(0.3)$ & $3 \times 10^{-6}$ & 0.9 \\
\hline ZKSCAN8 & rs13205911 & 6 & 28232093 & $\mathrm{~T}$ & 0.09 & $-2.5(0.5)$ & $5 \times 10^{-6}$ & 0.6 \\
\hline
\end{tabular}

Abbreviations: CA, coded allele; CAF, coded allele frequency; $\mathrm{Chr}$, chromosome; $P_{\text {het, }} P$-value of heterogeneity; SNP, single-nucleotide polymorphism. ${ }^{\mathrm{a} B u i l d} 36$ base-pair position.

heterogeneity, and examined QT as well as its component parts (QRS, JT), we did not identify any genome-wide significant SNPs modifying the association between thiazides and these ECG intervals. However, we identified 74 loci with suggestive evidence of association through either univariate or cross-phenotype analyses as well as evidence of enrichment in pathways involved in transcription and translation.

Interestingly, our suggestive results included multiple loci involved in ion transport and handling, the disruption of which is believed to be an underlying mechanism in drug-induced QT prolongation, ${ }^{66}$ supporting the hypothesis that common SNPs modify the thiazide-QT relationship. For example, the NELL1 locus was previously associated with changes in fasting plasma triglyceride levels in response to hydrochlorothiazide use. ${ }^{67}$ Other interesting suggestive results include the PITX2 and RYR3 QRS loci identified in Hispanic/Latinos, which may directly regulate ion channel genes and genes involved in calcium handling. ${ }^{68}$ Moreover, we found suggestive evidence of thiazide-SNP interactions on QT, QRS, or JT in other genes involved in ion transport and handling, including STC2, ${ }_{1}^{69} E D N 1_{1}^{70}$ TRPC7, ${ }^{71} P K P 2^{72}$ and $D I S C 1,{ }^{73}$ as well as a voltage-gated potassium channel gene (KCNQ3).

Despite these intriguing results, our power simulations suggested there was limited power to detect interaction effects of $2 \mathrm{~ms}$, sizes consistent with QT main effects analyses. ${ }^{65}$ The low power suggests that larger sample sizes and/or innovative statistical methods may be required to study gene-environment interactions given the stringent genome-wide significance threshold. ${ }^{74-76}$ Furthermore, our power simulations demonstrated insufficient power to detect interaction effects of $5 \mathrm{~ms}$ or less for less common SNPs $(M A F=5 \%)$. Therefore, future work should utilize larger sample sizes, particularly studies with longitudinal data, if available.

Another limitation of our work was that medication use data were collected infrequently, e.g. years apart. Particularly, medication assessments covered only one to two weeks of medication use in most participating cohorts and variables such as medication dosage and duration of use were not available universally across studies. Previous work has demonstrated a dose-dependent relationship between thiazide use and cardiac arrest, a potential outcome of QT prolongation. ${ }^{77}$ However, we were unable to identify participants using high dose thiazides because medication dosage data was unavailable in all cohorts. Furthermore, $\mathrm{K}^{+}$ measurements and information on $\mathrm{K}^{+}$supplements was not obtained across all cohorts so we were unable to adjust for $\mathrm{K}^{+}$ levels in our analyses, despite the known role of thiazide diuretics in inducing hypokalemia and the role of hypokalemia in causing QT prolongation. ${ }^{78,79}$

Furthermore, ECG intervals are known to vary in the presence of cardiovascular disease (CVD). ${ }^{80}$ While we did exclude participants with certain types of CVD including prevalent heart failure and atrial fibrillation, we were not able to further characterize the role 

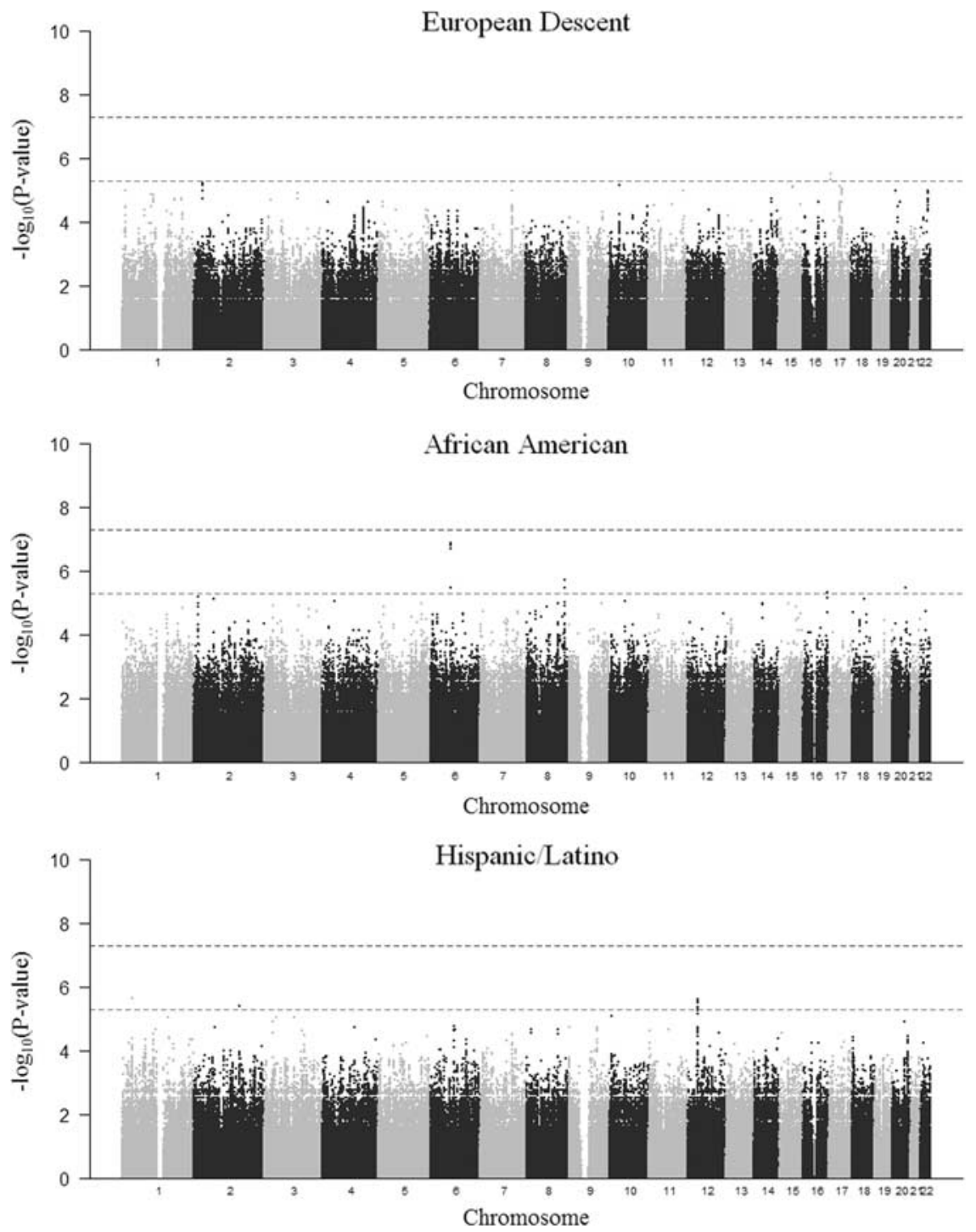

Figure 2. Manhattan plots of $P$-values thiazide-SNP interaction estimates after cross-phenotype meta-analysis (QRS interval, JT interval) using aSPU among European descent populations $(N=47836)$, African American populations $(N=11482)$, and Hispanic/Latino populations (15 116). For each trait separately, each study was analyzed using linear regression, mixed-effects models, or generalized estimating equations and SNPs with a study-specific degree of freedom measure ( $\mathrm{df}=$ twice the cohort- and SNP-specific product of the SNP imputation quality (range: 0,1$)$, the MAF (range: $0.0,0.50)$, and the number of individuals exposed to thiazide $\left.\left(N_{\text {exposed }}\right)\right)<15$ were excluded from cross-phenotype meta-analysis. The $x$-axis represents the chromosomal position and the $y$-axis represents the $-\log _{10}(P$-value). On each plot, genome-wide significance $\left(P<5 \times 10^{-8}\right)$ and suggestive significance $\left(P<5 \times 10^{-6}\right)$ are denoted with dashed lines.

of CVD in the pharmacogenomics of thiazide use and QT duration. Given that we saw larger mean QT and JT intervals in Hispanic/ Latino populations than in European descent or African American populations in our study sample, as well a substantial difference in mean exposure to thiazides, ranging from just $9 \%$ in Hispanic/ Latinos to $37 \%$ in African Americans, our analyses are limited by the heterogeneity of exposure and outcome in our population. The large difference in thiazide exposure between race/ethnic groups could also indicate an underlying difference in CVD prevalence among our populations. Considering that pharmacogenomic studies such as this one are already limited in their power to detect effects, the addition of unmeasured heterogeneity such as CVD status could further reduce our power to detect genetic effects modifying the relationship between thiazides and QT. Therefore, future work should consider alternate study designs, such as clinical trials or specially collected cohorts, as settings for pharmacogenomics work. In clinical trials or specialty cohorts, populations can be more closely controlled and therefore more homogeneous in traits that may confound the relationship between thiazides and QT.

Additionally, observational cohort studies are known to be susceptible to selection biases, such as prevalent user bias, whereby long-term medication users are least likely to suffer from ADRs and users with ADRs often stop therapy and therefore have a lower chance of being seen while on therapy. ${ }^{81,82}$ Unfortunately, without information on duration of use, it is difficult to evaluate the effect of prevalent user bias on study results. Indeed, it is unclear if these biases are of concern in pharmacogenomic studies. ${ }^{83,84}$ Additional work is needed to assess whether selection bias requires more consideration in pharmacogenomic research and to assess possible advantages of alternative designs, such as active comparator designs (whereby the control group contains participants using a different class of medications with similar indications to the medication of interest) or new user 
Table 3. Loci with suggestive evidence modifying the effect of Thiazide on QRS and JT intervals after cross-phenotype meta-analysis

\begin{tabular}{|c|c|c|c|c|c|c|c|c|}
\hline \multirow[t]{2}{*}{ Locus } & \multirow[t]{2}{*}{ SNP } & \multirow[t]{2}{*}{ Chr } & \multirow[t]{2}{*}{ Position $^{a}$} & \multirow[t]{2}{*}{$C A$} & \multirow[t]{2}{*}{$C A F$} & \multirow[t]{2}{*}{ P-value } & \multicolumn{2}{|c|}{ Univariate P-value } \\
\hline & & & & & & & QRS & $J T$ \\
\hline \multicolumn{9}{|c|}{ European descent } \\
\hline PIK3R6 & rs1295230 & 17 & 8682305 & $\mathrm{~T}$ & 0.02 & $3 \times 10^{-6}$ & 0.008 & 0.001 \\
\hline \multicolumn{9}{|c|}{ African American } \\
\hline ADGRB3 & rs6931354 & 6 & 69527128 & A & 0.21 & $1 \times 10^{-7}$ & 0.005 & 0.0002 \\
\hline$A D C Y 8$ & rs10108730 & 8 & 131767803 & $\mathrm{~T}$ & 0.79 & $2 \times 10^{-6}$ & $1 \times 10^{-5}$ & 0.0003 \\
\hline PREX1 & rs8119517 & 20 & 46464282 & A & 0.94 & $3 \times 10^{-6}$ & 0.0005 & 0.02 \\
\hline $\mathrm{CDH} 13$ & rs11649358 & 16 & 81415652 & A & 0.75 & $5 \times 10^{-6}$ & $9 \times 10^{-6}$ & 0.001 \\
\hline \multicolumn{9}{|c|}{ Hispanic/Latino } \\
\hline AK2 & rs11591185 & 1 & 33274771 & A & 0.07 & $2 \times 10^{-6}$ & $7 \times 10^{-7}$ & $3 \times 10^{-5}$ \\
\hline ASS1P14 & rs12578228 & 12 & 33030528 & $\mathrm{~T}$ & 0.10 & $2 \times 10^{-6}$ & $2 \times 10^{-6}$ & $2 \times 10^{-5}$ \\
\hline GALNT13 & rs17553946 & 2 & 155055407 & A & 0.23 & $4 \times 10^{-6}$ & 0.005 & $9 \times 10^{-7}$ \\
\hline
\end{tabular}

Abbreviations: CA, coded allele; CAF, coded allele frequency; Chr, chromosome; JT, JT interval; QRS, QRS interval; SNP, single-nucleotide polymorphism. ${ }^{\text {a Build }}$ 36 base-pair position.

\begin{tabular}{|c|c|c|c|c|}
\hline \multirow[t]{7}{*}{ QT } & \multirow[t]{5}{*}{ Hispanic/Latino } & Nucleotide Binding & $5 \times 10^{-6}$ & 0.004 \\
\hline & & tRNA Adenine-N1 Methyltransferase Activity & $6 \times 10^{-5}$ & 0.03 \\
\hline & & Transcription Coactivator Activity & $8 \times 10^{-5}$ & 0.03 \\
\hline & & Transcriptional Activity of SMAD2, SMAD3, SMAD4, Heterotrimer & 0.0001 & 0.03 \\
\hline & & Zinc lon Binding & 0.0002 & 0.04 \\
\hline & \multirow[t]{2}{*}{ Trans-Ethnic } & General RNA Polymerase II Transcription & $4 \times 10^{-6}$ & 0.006 \\
\hline & & Transcription & $4 \times 10^{-5}$ & 0.03 \\
\hline \multirow[t]{4}{*}{ JT } & \multirow[t]{4}{*}{ African American } & Transcription Factor TFIID Complex & $7 \times 10^{-5}$ & 0.05 \\
\hline & & Aminoacyl-tRNA Synthetase Multienzyme Complex & 0.0001 & 0.05 \\
\hline & & tRNA Aminoacylation for Protein Translation & 0.0001 & 0.05 \\
\hline & & Transcription Factor TFTC Complex & 0.0001 & 0.05 \\
\hline
\end{tabular}

Abbreviations: FDR, false discovery rate; JT, JT interval; QT, QT interval.

designs (whereby prevalent users are excluded). Moreover, medication inventories may be associated with non-negligible measurement error. For example, while Smith et al. reported good agreement between thiazide use measured using medication inventories and serum thiazide measurements, specificity remained moderate. ${ }^{85}$

Given the challenges associated with assembling an adequately powered pharmacogenomics study, electronic medical records (EMRs) represent a potential untapped resource that may merit evaluation. Strengths of EMRs include the potential to provide a more complete medication history, which could enable sensitivity analyses examining variables such as medication dose and duration of use. Furthermore, consortia such as eMERGE have demonstrated the feasibility of linking EMRs to genetic data for use in genetic research, ${ }^{86}$ and have successfully identified genetic variants modifying drug response. ${ }^{87}$ However, EMRs have limitations. Investigators using EMR data cannot control participant recruitment, timing and accuracy of data collection, or population representativeness. ${ }^{88}$ Considering ECG research specifically, cohort studies administer ECGs to all participants at study visits, whereas
EMRs may capture ECGs for patients with medical indications, providing an inherently different population. EMRs therefore have the potential to greatly advance pharmacogenomic research but warrant further evaluation.

In conclusion, our findings suggest that additional work is needed to fully elucidate potential pharmacogenomic effects influencing the thiazide-QT relationship. Our suggestive results support a possible role of genetics in modifying the association between thiazides and QT. However, these findings can inform the biology of thiazide-induced QT prolongation and do not preclude the possibility of common variants with small effects or rare variants with larger effects. Future work that leverages larger sample sizes, such as those available in EMRs, and innovative statistical methods to validate these suggestive findings is needed. The FDA considers further regulation of drugs that prolong QT by as little as $5 \mathrm{~ms}$, a small increment easily achieved by the combination of genetic and pharmaceutical effects, ${ }^{37,89}$ making it critical that we unravel the complex etiology of drug-induced QT prolongation. ${ }^{90}$ Pharmacogenomics remain a promising avenue for understanding variability in drug 


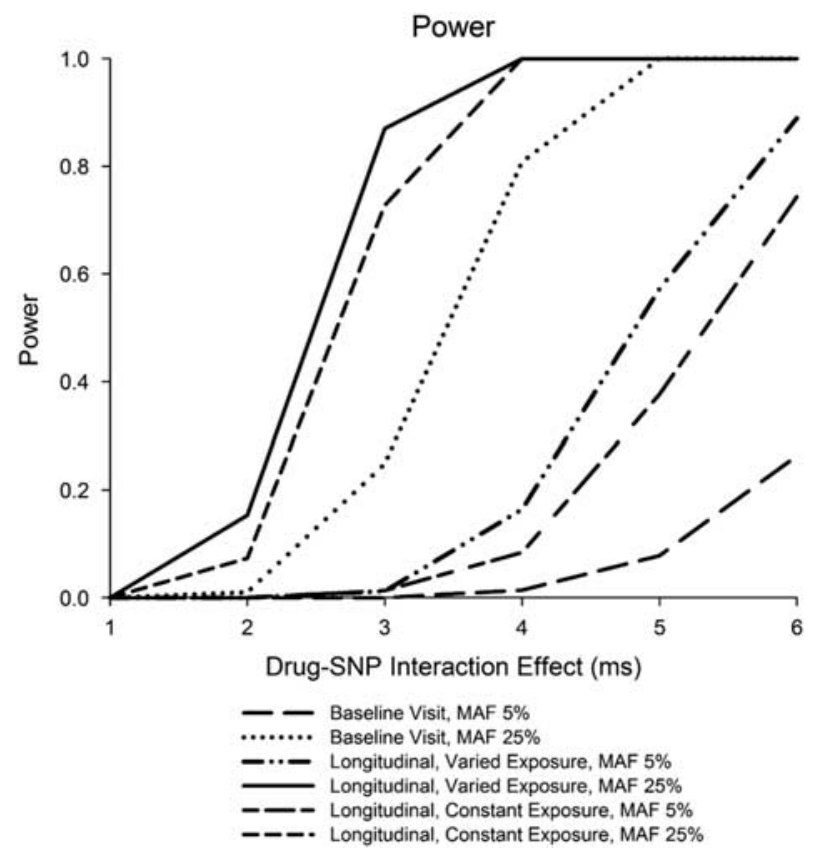

Figure 3. Statistical power of a simulated pharmacogenomics study of QT. The $x$-axis represents the range of tested drug-SNP interaction effects in milliseconds (ms). The $y$-axis represents the power to detect the tested drug-SNP interaction effect. The following assumptions were used for the calculations: 2 serial visits measuring electrocardiograms (ECGs) and drug exposure, $N=50000$ participants, a single-nucleotide polymorphism (SNP) minor allele frequency (MAF) of $5 \%$ or $25 \%$, and the $N_{\text {exposed }}=8100$. Simulation analyses were run using only the baseline visit (cross-sectional) and a longitudinal model. Under the longitudinal model, simulations were run with all participants having constant drug exposure across visits or having varied drug exposure across visits. Cross-sectional models were run using linear regression and longitudinal models were run using a generalized estimating equation with an independence working correlation.

response and for utilizing genetics to improve public health but innovative solutions are needed to overcome inherent challenges.

\section{CONFLICT OF INTEREST}

The authors declare no conflict of interest.

\section{ACKNOWLEDGMENTS}

Age, Gene/Environment Susceptibility-Reykjavik Study (AGES): This study has been funded by NIH contracts N01-AG-1-2100 and 271201200022C, the NIA Intramural Research Program, Hjartavernd (the Icelandic Heart Association), and the Althingi (the Icelandic Parliament). The study is approved by the Icelandic National Bioethics Committee, VSN: 00-063. The researchers are indebted to the participants for their willingness to participate in the study.

Atherosclerosis Risk in Communities (ARIC): The Atherosclerosis Risk in Communities Study is carried out as a collaborative study supported by National Heart, Lung and Blood Institute Contracts (HHSN268201100005C, HHSN268201100006C, HHSN268201100007C, HHSN268201100008C, HHSN268201100009C, HHSN268 201100010C, HHSN268201100011C and HHSN268201100012C), R01HL087641, R01HL59367 and R01HL086694; National Human Genome Research Institute Contract U01HG004402; and National Institutes of Health Contract HHSN268200625226C. We thank the staff and participants of the ARIC study for their important contributions. Infrastructure was partly supported by Grant no. UL1RR025005, a component of the National Institutes of Health and NIH Roadmap for Medical Research. AAS was supported by NHLBI Training grants T32HL7055 and T32HL07779.

Cardiovascular Health Study (CHS): This CHS research was supported by NHLBI contracts HHSN268201200036C, HHSN268200800007C, N01HC55222, N01HC85079, N01HC85080, N01HC85081, N01HC85082, N01HC85083, N01HC85086; and NHLBI grants U01HL080295, R01HL087652, R01HL105756, R01HL103612, R01HL120393, HL130114, and R01HL085251with additional contribution from the National Institute of Neurological Disorders and Stroke (NINDS). Additional support was provided through R01AG023629 from the National Institute on Aging (NIA). A full list of principal CHS investigators and institutions can be found at CHS-NHLBl.org. The provision of genotyping data was supported in part by the National Center for Advancing Translational Sciences, CTSI grant UL1TR000124, and the National Institute of Diabetes and Digestive and Kidney Disease Diabetes Research Center (DRC) grant DK063491 to the Southern California Diabetes Endocrinology Research Center. NS was supported by R01HL116747 and RO1HL111089.

Erasmus Rucphen Family Study (ERF): The ERF study, as a part of EUROSPAN (European Special Populations Research Network), was supported by European Commission FP6 STRP grant number 018947 (LSHG-CT-2006-01947) and also received funding from the European Community's Seventh Framework Programme (FP7/2007-2013)/grant agreement HEALTH-F4-2007-201413 by the European Commission under the programme 'Quality of Life and Management of the Living Resources' of 5th Framework Programme (no. QLG2-CT-2002-01254). The ERF study was further supported by ENGAGE consortium and CMSB. High-throughput analysis of the ERF data was supported by joint grant from Netherlands Organisation for Scientific Research and the Russian Foundation for Basic Research (NWO-RFBR 047.017.043). Exome sequencing in ERF was supported by the ZonMw grant (project 91111025). We are grateful to all study participants and their relatives, general practitioners and neurologists for their contributions to the ERF study and to P. Veraart for her help in genealogy, J. Vergeer for the supervision of the laboratory work and P. Snijders for his help in data collection.

Framingham Heart Study (FHS): FHS work was supported by the National Heart Lung and Blood Institute of the National Institutes of Health and Boston University School of Medicine (Contract No. N01-HC-25195 and Contract No. HHSN268201500001I), its contract with Affymetrix for genotyping services (Contract No. N02-HL-6-4278), based on analyses by FHS investigators participating in the SNP Health Association Resource (SHARe) project. A portion of this research was conducted using the Linux Cluster for Genetic Analysis (LinGA-II), funded by the Robert Dawson Evans Endowment of the Department of Medicine at Boston University School of Medicine and Boston Medical Center. Measurement of the Gen 3 ECGs was supported by grants from the Doris Duke Charitable Foundation and the Burroughs Wellcome Fund (Newton-Cheh) and the NIH (HL080025, Newton-Cheh).

Health 2000: Supported by the Orion-Farmos Research Foundation (KK and KP), the Finnish Foundation for Cardiovascular Research (KK, KP) and the Academy of Finland (Grant Nos. 129494 and 139635 to VS).

Health, Aging, and Body Composition (Health $A B C$ ): This research was supported by NIA Contracts N01AG62101, N01AG62103 and N01AG62106. The genome-wide association study was funded by NIA Grant 1R01AG032098-01A1 to Wake Forest University Health Sciences and genotyping services were provided by the Center for Inherited Disease Research (CIDR). CIDR is fully funded through a federal contract from the National Institutes of Health to The Johns Hopkins University, Contract No. HHSN268200782096C. This research was supported in part by the Intramural Research Program of the $\mathrm{NIH}$, National Institute on Aging.

Hispanic Community Health Study/Study of Latinos (HCHS/SOL): We thank the participants and staff of the $\mathrm{HCHS} / \mathrm{SOL}$ study for their contributions to this study. The baseline examination of $\mathrm{HCHS} / \mathrm{SOL}$ was carried out as a collaborative study supported by contracts from the National Heart, Lung, and Blood Institute (NHLBI) to the University of North Carolina (N01-HC65233), University of Miami (N01HC65234), Albert Einstein College of Medicine (N01-HC65235), Northwestern University (N01-HC65236), and San Diego State University (N01-HC65237). The following Institutes/Centers/Offices contributed to the first phase of $\mathrm{HCHS} / \mathrm{SOL}$ through a transfer of funds to the NHLBI: National Institute on Minority Health and Health Disparities, National Institute on Deafness and Other Communication Disorders, National Institute of Dental and Craniofacial Research (NIDCR), National Institute of Diabetes and Digestive and Kidney Diseases, National Institute of Neurological Disorders and Stroke, NIH Institution-Office of Dietary Supplements. The Genetic Analysis Center at University of Washington was supported by NHLBI and NIDCR contracts (HHSN268201300005C AM03 and MOD03). Genotyping efforts were supported by NHLBI HSN 26220/20054C, NCATS CTSI grant UL1TR000124, and NIDDK Diabetes Research Center (DRC) grant DK063491.

Jackson Heart Study (JHS): We thank the Jackson Heart Study (JHS) participants and staff for their contributions to this work. The JHS is supported by contracts HHSN268201300046C, HHSN268201300047C, HSN268201300048C, HHSN268201300 049C, HHSN268201300050C from the National Heart, Lung, and Blood Institute and the National Institute on Minority Health and Health Disparities.

Multi-Ethnic Study of Atherosclerosis (MESA): MESA and MESA SNP Health Association Resource (SHARe) are conducted and supported by the National Heart, Lung and Blood Institute (NHLBI) in collaboration with MESA investigators. Support is provided by grants and contracts N01-HC-95159, N01-HC-95160, N01-HC-95161, N01-HC-95162, N01-HC-95163, N01-HC-95164, N01-HC-95165, N01-HC-95166, N01HC-95167, N01-HC-95168, N01-HC-95169 and RR-024156. Additional funding was 
supported in part by the Clinical Translational Science Institute grant UL1RR033176 and is now at the National Center for Advancing Translational Sciences, CTSI grant UL1TR000124. We also thank the other investigators, the staff and the participants of the MESA study for their valuable contributions. A full list of participating MESA investigators and institutions can be found at http://www.mesa-nhlbi.org.

The Netherlands Epidemiology of Obesity (NEO): The authors of the NEO study thank all individuals who participated in the Netherlands Epidemiology in Obesity study, all participating general practitioners for inviting eligible participants and all research nurses for collection of the data. We thank the NEO study group, Pat van Beelen, Petra Noordijk and Ingeborg de Jonge for the coordination, lab and data management of the NEO study. The genotyping in the NEO study was supported by the Centre National de Génotypage (Paris, France), headed by Jean-Francois Deleuze. The NEO study is supported by the participating Departments, the Division and the Board of Directors of the Leiden University Medical Center, and by the Leiden University, Research Profile Area Vascular and Regenerative Medicine. Dennis Mook-Kanamori is supported by Dutch Science Organization (ZonMW-VENI Grant 916.14.023).

Prospective Study of Pravastatin in the Elderly at Risk (PROSPER): The PROSPER study was supported by an investigator initiated grant obtained from Bristol-Myers Squibb. Professor Dr J W Jukema is an Established Clinical Investigator of the Netherlands Heart Foundation (Grant no. 2001 D 032). Support for genotyping was provided by the seventh framework program of the European commission (Grant no. 223004) and by the Netherlands Genomics Initiative (Netherlands Consortium for Healthy Aging Grant 050-060-810).

Rotterdam Study (RS): The RS is supported by the Erasmus Medical Center and Erasmus University Rotterdam; The Netherlands Organization for Scientific Research; The Netherlands Organization for Health Research and Development (ZonMw); the Research Institute for Diseases in the Elderly; The Netherlands Heart Foundation; the Ministry of Education, Culture and Science; the Ministry of Health Welfare and Sports; the European Commission; and the Municipality of Rotterdam. Support for genotyping was provided by The Netherlands Organization for Scientific Research (NWO) (175.010.2005.011, 911.03.012) and Research Institute for Diseases in the Elderly (RIDE). This study was supported by The Netherlands Genomics Initiative (NGI)/Netherlands Organization for Scientific Research (NWO) Project No. 050-060810. This collaborative effort was supported by an award from the National Heart, Lung and Blood Institute (R01-HL-103612, PI BMP). CLA was supported in part by Grant R00-HL-098458 from the National Heart, Lung, and Blood Institute.

Women's Health Initiative Clinical Trial (WHI CT): The Women's Health Initiative clinical trials were funded by the National Heart, Lung, and Blood Institute, National Institutes of Health, U.S. Department of Health and Human Services through contracts HHSN268201100046C, HHSN268201100001C, HHSN268201100002C, HHSN26820110 0003C, HHSN268201100004C, and HHSN271201100004C. All contributors to WHI science are listed at https://www.whi.org/researchers/Documents\%20\%20Write\%20a \%20Paper/WHI\%20Investigator\%20Long\%20List.pdf. ELB was supported in part by a grant from the National Cancer Institute (5T32CA009001). WHI GARNET: Within the Genomics and Randomized Trials Network, a GWAS of Hormone Treatment and CVD and Metabolic Outcomes in the WHI was funded by the National Human Genome Research Institute, National Institutes of Health, U.S. Department of Health and Human Services through cooperative agreement U01HG005152 (Reiner). All contributors to GARNET science are listed at https://www.garnetstudy.org/Home. WHI MOPMAP: The Modification of PM-Mediated Arrhythmogenesis in Populations was funded by the National Institute of Environmental Health Sciences, National Institutes of Health, U.S. Department of Health and Human Services through grant R01ES017794 (Whitsel). WHI SHARe: The SNP Health Association Resource project was funded by the National Heart, Lung and Blood Institute, National Institutes of Health, U.S. Department of Health and Human Services through contract N02HL64278 (Kooperberg). WHI WHIMS: The Women's Health Initiative Memory Study (WHIMS+) Genome-Wide Association Study was funded by the National Heart, Lung, and Blood Institute, National Institutes of Health, U.S. Department of Health and Human Services through contract HHSN268201100046C (Anderson).

\section{DISCLAIMER}

The views expressed in this manuscript are those of the authors and do not necessarily represent the views of the National Heart, Lung, and Blood Institute; the National Institutes of Health; or the U.S. Department of Health and Human Services.

\section{REFERENCES}

$1 \mathrm{Gu}$ Q, Dillon CF, Burt VL. Prescription drug use continues to increase: U.S. prescription drug data for 2007-2008. NCHS Data Brief 2010; 42: 1-8.

2 Abdessadek M, Magoul R, Amarti A, El Ouezzani S, Khabbal Y. Customizing dosage drugs what contribution in therapeutic drug monitoring? Ann Biol Clin 2014; 72: $15-24$
3 El Desoky ES, Derendorf H, Klotz U. Variability in response to cardiovascular drugs. Curr Clin Pharmacol 2006; 1: 35-46.

4 Thummel KE, Lin YS. Sources of interindividual variability. Methods Mol Biol 2014; 1113: 363-415.

5 Zhang Y, Post WS, Dalal D, Blasco-Colmenares E, Tomaselli GF, Guallar E. QT-interval duration and mortality rate: results from the Third National Health and Nutrition Examination Survey. Arch Intern Med 2011; 171: 1727-1733.

6 Arizona Center for Education and Research on Therapeutics. QTDrugs Lists. Available at https://www.crediblemeds.org/. Accessed November 17, 2014.

7 Murphy JG, Lloyd MA. Mayo Clinic Cardiology Concise Textbook and Mayo Clinic Cardiology Board Review Questions \& Answers: (TEXT AND Q\&A SET). Taylor \& Francis: Boca Raton, FL, 2007.

8 Roden DM. Drug-Induced prolongation of the QT interval. N Engl J Med 2004; 350: 1013-1022.

9 Al-Khatib SM, LaPointe NMA, Kramer JM, Califf RM. What clinicians should know about the QT interval. JAMA 2003; 289: 2120-2127.

10 Zipes DP, Jalife J. Cardiac Electrophysiology: From Cell to Bedside 4th Edition ed. Elsevier Inc: Philadelphia, 2004.

11 Lee JW, Aminkeng F, Bhavsar AP, Shaw K, Carleton BC, Hayden MR et al. The emerging era of pharmacogenomics: current successes, future potential, and challenges. Clin Genet 2014; 86: 21-28.

12 Khoury MJ, Gwinn M, Clyne M, Yu W. Genetic epidemiology with a capital E, ten years after. Genet Epidemiol 2011; 35: 845-852.

13 Puri A, Saif MW. Pharmacogenomics update in pancreatic cancer. JOP 2014; 15: 114-117.

14 Weitzel KW, Elsey AR, Langaee TY, Burkley B, Nessl DR, Obeng AO et al. Clinical pharmacogenetics implementation: Approaches, successes, and challenges. Am J Med Genet 2014; 166: 56-67.

15 Aminkeng F. Using pharmacogenetics in real time to guide therapy: the warfarin example. Clin Genet 2014; 85: 533-534.

16 Daneshjou R, Tatonetti NP, Karczewski KJ, Sagreiya H, Bourgeois S, Drozda K et al. Pathway analysis of genome-wide data improves warfarin dose prediction. BMC Genomics 2013; 14: S11.

17 Jonas DE, Wines R. Pharmacogenomic testing and the prospect of individualized treatment. N C Med J 2013; 74: 485-493.

18 Niinuma $Y$, Saito T, Takahashi M, Tsukada C, Ito M, Hirasawa N et al. Functional characterization of 32 CYP2C9 allelic variants. Pharmacogenomics J 2014; 14: 107-114.

19 Perera MA, Cavallari LH, Limdi NA, Gamazon ER, Konkashbaev A, Daneshjou $R$ et al. Genetic variants associated with warfarin dose in AfricanAmerican individuals: a genome-wide association study. Lancet 2013; 382: 790-796.

20 Fellay J, Thompson AJ, Ge D, Gumbs CE, Urban TJ, Shianna KV et al. ITPA gene variants protect against anaemia in patients treated for chronic hepatitis C. Nature 2010; 464: 405-408.

21 Phillips KA, Veenstra DL, Oren E, Lee JK, Sadee W. Potential role of pharmacogenomics in reducing adverse drug reactions: a systematic review. JAMA 2001; 286: 2270-2279.

22 Wilke RA, Dolan ME. Genetics and variable drug response. JAMA 2011; 306: 306-307.

23 Akylbekova EL, Crow RS, Johnson WD, Buxbaum SG, Njemanze S, Fox E et al. Clinical correlates and heritability of QT interval duration in blacks: the Jackson Heart Study. Circ Arrhythm Electrophysiol 2009; 2: 427-432.

24 Carter N, Snieder H, Jeffery S, Saumarez R, Varma C, Antoniades L et al. QT interval in twins. J Hum Hypertens 2000; 14: 389-390.

25 Hanson B, Tuna N, Bouchard T, Heston L, Eckert E, Lykken D et al. Genetic factors in the electrocardiogram and heart rate of twins reared apart and together. $\mathrm{Am} \mathrm{J}$ Cardiol 1989; 63: 606-609.

26 Lehtinen AB, Newton-Cheh C, Ziegler JT, Langefeld CD, Freedman BI, Daniel KR et al. Association of NOS1AP genetic variants with QT interval duration in families from the diabetes heart study. Diabetes 2008; 57: 1108-1114.

27 Silva $\mathrm{CT}$, Kors JA, Amin N, Dehghan A, Witteman JC, Willemsen R et al. Heritabilities, proportions of heritabilities explained by GWAS findings, and implications of cross-phenotype effects on PR interval. Hum Genet 2015; 134: 1211-1219.

28 Gu Q, Burt VL, Dillon CF, Yoon S. Trends in antihypertensive medication use and blood pressure control among United States adults with hypertension: the National Health And Nutrition Examination Survey, 2001 to 2010. Circulation 2012; 126: 2105-2114.

29 Duarte JD, Turner ST, Tran B, Chapman AB, Bailey KR, Gong Y et al. Association of chromosome 12 locus with antihypertensive response to hydrochlorothiazide may involve differential YEATS4 expression. Pharmacogenom J 2013; 13: 257-263.

30 Li Y, Yang P, Wu SL, Yuan JX, Wu Y, Zhao DD et al. [Effect of CYP11B2 gene -344T/C polymorphism on renin-angiotensin-aldosterone system activity and blood 
pressure response to hydrochlorothiazide]. Zhonghua yi xue yi chuan xue za zhi 2012; 29: 68-71.

31 Li Y, Zhou Y, Yang P, Niu JQ, Wu Y, Zhao DD et al. Interaction of ACE and CYP11B2 genes on blood pressure response to hydrochlorothiazide in Han Chinese hypertensive patients. Clin Exp Hypertens 2011; 33: 141-146.

32 McDonough CW, Burbage SE, Duarte JD, Gong Y, Langaee TY, Turner ST et al. Association of variants in NEDD4L with blood pressure response and adverse cardiovascular outcomes in hypertensive patients treated with thiazide diuretics. J Hypertens 2013; 31: 698-704.

33 Turner ST, Bailey KR, Fridley BL, Chapman AB, Schwartz GL, Chai HS et al. Genomic association analysis suggests chromosome 12 locus influencing antihypertensive response to thiazide diuretic. Hypertension 2008; 52: 359-365.

34 Turner ST, Boerwinkle E, O'Connell JR, Bailey KR, Gong Y, Chapman AB et al. Genomic association analysis of common variants influencing antihypertensive response to hydrochlorothiazide. Hypertension 2013; 62: 391-397.

35 Centers for Disease Control Prevention. Vital signs: prevalence, treatment, and control of hypertension--United States, 1999-2002 and 2005-2008. MMWR 2011; 60: 103-108.

36 Go AS, Mozaffarian D, Roger VL, Benjamin EJ, Berry JD, Borden WB et al. Heart disease and stroke statistics-2013 update: a report from the American Heart Association. Circulation 2013; 127: e6-e245.

37 Avery $\mathrm{CL}$, Sitlani CM, Arking DE, Arnett DK, Bis JC, Boerwinkle E et al. Drug-gene interactions and the search for missing heritability: a cross-sectional pharmacogenomics study of the QT interval. Pharmacogenom J 2014; 14: 6-13.

38 Psaty BM, O'Donnell CJ, Gudnason V, Lunetta KL, Folsom AR, Rotter Jl et al. Cohorts for Heart and Aging Research in Genomic Epidemiology (CHARGE) Consortium: design of prospective meta-analyses of genome-wide association studies from 5 cohorts. Circ Cardiovasc Genet 2009; 2: 73-80.

39 Sitlani CM, Rice KM, Lumley T, McKnight B, Cupples LA, Avery CL et al. Generalized estimating equations for genome-wide association studies using longitudinal phenotype data. Stat Med 2014; 34: 118-130.

40 International HapMap Consortium. The International HapMap Project. Nature 2003; 426: 789-796.

41 International HapMap Consortium. A haplotype map of the human genome. Nature 2005; 437: 1299-1320.

42 International HapMap Consortium, Altshuler DM, Gibbs RA, Peltonen L, Altshuler DM, Gibbs RA et al. Integrating common and rare genetic variation in diverse human populations. Nature 2010; 467: 52-58.

43 The 1000 Genomes Project Consortium. A map of human genome variation from population-scale sequencing. Nature 2010; 467: 1061-1073.

44 The 1000 Genomes Project Consortium. An integrated map of genetic variation from 1,092 human genomes. Nature 2012; 491: 56-65.

45 Kent WJ, Sugnet CW, Furey TS, Roskin KM, Pringle TH, Zahler AM et al. The Human Genome Browser at UCSC. Genome Res 2002; 12: 996-1006.

46 UCSC Human Genome Browser Lift Genome Annotations. Available at http:// genome.ucsc.edu/cgi-bin/hgLiftOver.

47 Satterthwaite FE. An approximate distribution of estimates of variance components. Biometrics 1946; 2: 110-114.

48 Willer CJ, Li Y, Abecasis GR. METAL: fast and efficient meta-analysis of genomewide association scans. Bioinformatics 2010; 26: 2190-2191.

49 Ramos E, Doumatey A, Elkahloun AG, Shriner D, Huang H, Chen G et al. Pharmacogenomics, ancestry and clinical decision making for global populations. Pharmacogenom J 2013; 14: 217-222.

50 Thomas D. Gene-environment-wide association studies: emerging approaches. Nat Rev Genet 2010; 11: 259-272.

51 Morris AP. Transethnic meta-analysis of genomewide association studies. Genet Epidemiol 2011; 35: 809-822.

52 Bolormaa S, Pryce JE, Reverter A, Zhang Y, Barendse W, Kemper K et al. A multitrait, meta-analysis for detecting pleiotropic polymorphisms for stature, fatness and reproduction in beef cattle. PLoS Genet 2014; 10: e1004198.

53 Chung D, Yang C, Li C, Gelernter J, Zhao H. GPA: a statistical approach to prioritizing GWAS results by integrating pleiotropy and annotation. PLoS Genet 2014; 10: e1004787.

54 Kim J, Bai Y, Pan W. An adaptive association test for multiple phenotypes with GWAS summary statistics. Genet Epidemiol 2015; 39: 651-663.

55 Gui H, Li M, Sham PC, Cherny SS. Comparisons of seven algorithms for pathway analysis using the WTCCC Crohn's disease dataset. BMC Res Notes 2011; 4: 386.

56 The Network Pathway Analysis Subgroup of the Psychiatric Genomics Consortium. Psychiatric genome-wide association study analyses implicate neuronal, immune and histone pathways. Nat Neurosci 2015; 18: 199-209.

57 Väremo L, Nielsen J, Nookaew I. Enriching the gene set analysis of genome-wide data by incorporating directionality of gene expression and combining statistical hypotheses and methods. Nucleic Acids Res 2013; 41: 4378-4391.

58 de Leeuw CA, Mooij JM, Heskes T, Posthuma D. MAGMA: generalized gene-set analysis of GWAS data. PLoS Comput Biol 2015; 11: e1004219.
59 Krämer A, Green J, Pollard J, Tugendreich S. Causal analysis approaches in ingenuity pathway analysis. Bioinformatics 2014; 30: 523-530.

$60 \mathrm{Mi} \mathrm{H}$, Thomas P. PANTHER pathway: an ontology-based pathway database coupled with data analysis tools. Methods Mol Biol 2009; 563: 123-140.

61 Kanehisa M, Goto S, Sato Y, Furumichi M, Tanabe M. KEGG for integration and interpretation of large-scale molecular data sets. Nucleic Acids Res 2012; 40: D109-D114.

62 Kamburov A, Pentchev K, Galicka H, Wierling C, Lehrach H, Herwig R. ConsensusPathDB: toward a more complete picture of cell biology. Nucleic acids Res 2011; 39: D712-D717.

63 Kamburov A, Wierling C, Lehrach H, Herwig R. ConsensusPathDB--a database for integrating human functional interaction networks. Nucleic Acids Res 2009; 37: D623-D628.

64 Pers TH, Karjalainen JM, Chan Y, Westra H-J, Wood AR, Yang J et al. Biological interpretation of genome-wide association studies using predicted gene functions. Nat Commun 2015; 6: 5890.

65 Arking DE, Pulit SL, Crotti L, van der Harst P, Munroe PB, Koopmann TT et al. Genetic association study of QT interval highlights role for calcium signaling pathways in myocardial repolarization. Nat Genet 2014; 46: 826-836.

66 Trinkley KE, Page RL 2nd, Lien H, Yamanouye K, Tisdale JE. QT interval prolongation and the risk of torsades de pointes: essentials for clinicians. Curr Med Res Opin 2013; 29: 1719-1726.

67 Del-Aguila JL, Beitelshees AL, Cooper-Dehoff RM, Chapman AB, Gums JG, Bailey K et al. Genome-wide association analyses suggest NELL1 influences adverse metabolic response to HCTZ in African Americans. Pharmacogenom J 2014; 14: 35-40.

68 Tao Y, Zhang M, Li L, Bai Y, Zhou Y, Moon AM et al. Pitx2, an atrial fibrillation predisposition gene, directly regulates ion transport and intercalated disc genes. Circ Cardiovasc Genet 2014; 7: 23-32.

69 Zeiger W, Ito D, Swetlik C, Oh-hora M, Villereal ML, Thinakaran G. Stanniocalcin 2 is a negative modulator of store-operated calcium entry. Mol Cell Biol 2011; 31: 3710-3722.

70 Bkaily G, Avedanian L, Al-Khoury J, Chamoun M, Semaan R, Jubinville-Leblanc C et al. Nuclear membrane R-type calcium channels mediate cytosolic ET-1-induced increase of nuclear calcium in human vascular smooth muscle cells. Can J Physiol Pharmacol 2015; 93: 291-297.

71 de Souza LB, Ambudkar IS. Trafficking mechanisms and regulation of TRPC channels. Cell Calcium 2014; 56: 43-50.

72 Cerrone M, Lin X, Zhang M, Agullo-Pascual E, Pfenniger A, Chkourko Gusky H et al. Missense mutations in plakophilin-2 cause sodium current deficit and associate with a Brugada syndrome phenotype. Circulation 2014; 129: 1092-1103.

73 Park SJ, Jeong J, Park YU, Park KS, Lee H, Lee N et al. Disrupted-in-schizophrenia-1 (DISC1) regulates endoplasmic reticulum calcium dynamics. Sci Rep 2015; 5: 8694.

74 Uher R. Gene-environment interactions in common mental disorders: an update and strategy for a genome-wide search. Soc Psychiatry Psychiatr Epidemiol 2014; 49: 3-14.

75 Franks PW, Pearson E, Florez JC. Gene-environment and gene-treatment interactions in type 2 diabetes: progress, pitfalls, and prospects. Diabetes Care 2013; 36: 1413-1421.

76 Fan R, Huang $\mathrm{CH}$, Hu I, Wang H, Zheng T, Lo SH. A partition-based approach to identify gene-environment interactions in genome wide association studies. BMC Proc 2014; 8: S60.

77 Siscovick DS, Raghunathan TE, Psaty BM, Koepsell TD, Wicklund KG, Lin X et al. Diuretic therapy for hypertension and the risk of primary cardiac arrest. $N$ Engl $J$ Med 1994; 330: 1852-1857.

78 Rautaharju PM, Manolio TA, Psaty BM, Borhani NO, Furberg CD. Correlates of QT prolongation in older adults (the Cardiovascular Health Study). Cardiovascular Health Study Collaborative Research Group. Am J Cardiol 1994; 73: 999-1002.

79 Tamargo J, Segura J, Ruilope LM. Diuretics in the treatment of hypertension. Part 1: thiazide and thiazide-like diuretics. Exp Opin Pharmacother 2014; 15: 527-547.

80 Ramirez AH, Schildcrout JS, Blakemore DL, Masys DR, Pulley JM, Basford MA et al. Modulators of normal ECG intervals identified in a large electronic medical record. Heart Rhythm 2011; 8: 271-277.

81 Choi HK, Nguyen US, Niu J, Danaei G, Zhang Y. Selection bias in rheumatic disease research. Nat Rev Rheumatol 2014; 10: 403-412.

82 Hudson M, Suissa S. Methodological Issues Relevant to Observational Studies, Registries, and Administrative Health Databases in RheumatologyUnderstanding Evidence-Based Rheumatology. Springer: New York, NY, pp 209-2282014.

83 Hunter DJ. Gene-environment interactions in human diseases. Nat Rev Genet 2005; 6: 287-298.

84 Morimoto LM, White E, Newcomb PA. Selection bias in the assessment of gene-environment interaction in case-control studies. Am J Epidemiol 2003; 158: 259-263. 
85 Smith NL, Psaty BM, Heckbert SR, Tracy RP, Cornell ES. The reliability of medication inventory methods compared to serum levels of cardiovascular drugs in the elderly. J Clin Epidemiol 1999; 52: 143-146.

86 Kho AN, Pacheco JA, Peissig PL, Rasmussen L, Newton KM, Weston $\mathrm{N}$ et al. Electronic medical records for genetic research: results of the eMERGE consortium. Sci Transl Med 2011; 3: 79re1.

87 Birdwell KA, Grady B, Choi L, Xu H, Bian A, Denny JC et al. The use of a DNA biobank linked to electronic medical records to characterize pharmacogenomic predictors of tacrolimus dose requirement in kidney transplant recipients. Pharmacogenet Genomics 2012; 22: 32-42.

88 Schneeweiss S, Avorn J. A review of uses of health care utilization databases for epidemiologic research on therapeutics. J Clin Epidemiol 2005; 58: 323-337.

89 Iribarren C, Round AD, Peng JA, Lu M, Zaroff JG, Holve TJ et al. Validation of a population-based method to assess drug-induced alterations in the QT interval: a self-controlled crossover study. Pharmacoepidemiol Drug Safety 2013; 22: $1222-1232$.

90 FDA. Guidance for Industry: E14 Clinical Evaluation of QT/QTc Interval Prolongation and Proarrhythmic Potential for Non-Antiarrhythmic Drugs. In: Services DoHaH, editor. 2005.

Supplementary Information accompanies the paper on the The Pharmacogenomics Journal website (http://www.nature.com/tpj) 